\title{
The relationship between osteoclastogenic and anti-osteoclastogenic pro-inflammatory cytokines differs in human osteoporotic and osteoarthritic bone tissues
}

\author{
Janja Zupan ${ }^{1 *}$, Radko Komadina ${ }^{2}$ and Janja Marc ${ }^{1}$
}

\begin{abstract}
Background: Pro-inflammatory cytokines possess osteoclastogenic or anti-osteoclastogenic activities. They influence osteoclasts directly or via the receptor activator of nuclear factor $\kappa \mathrm{B}$ (RANK), RANK ligand (RANKL) and osteoprotegerin (OPG) system. Recent evidence suggests that inflammation may play a role in osteoporosis (OP) and osteoarthritis $(\mathrm{OA})$. We aimed therefore to determine whether there is a difference between both groups: first, in the expression of the osteoclastogenic and anti-osteoclastogenic cytokines, second, in correlation of these cytokines with bone mineral density (BMD) and levels of bone turnover markers (BTM) and third, in correlation between the expression of these cytokines and osteoclast specific genes and RANK/RANKL/OPG genes.

Methods: Human bone samples from 54 age and sex matched patients with OP or OA were collected during hip arthroplasty surgery. The expression of 25 genes encoding pro-inflammatory cytokines, their receptors, osteoclast specific genes and RANK/RANKL/OPG genes was measured using quantitative real-time PCR. Total hip, femoral neck and lumbar spine BMD and BTM in blood samples were measured. The comparison between OP and OA was assessed using Student's t-test or Mann-Whitney $U$ test and correlations between gene expression, BMD and BTM were determined using nonparametric correlation.

Results: The results demonstrated a higher expression of interleukin (IL)- 6 and IL-1 $\alpha$ in OP, and interferon (IFN)- $\gamma$ in OA $(p<0.0005)$. Negative correlations of total hip BMD with tumor necrosis factor- $\alpha$ (TNF- $\alpha$ ) in OA and with RANKL/RANK in OP were found $(p<0.05)$. Significant correlations with BTM were shown for IL-1 $\alpha$ and IFN- $\gamma$ in OP (rho $=0.608$ and -0.634 ) and for TNF- $\alpha$, IL- 6 and transforming growth factor- $\beta 1$ (TGF- $\beta 1$ ) in OA (rho $=0.591,-0.521$ and 0.636). Results showed OP specific negative correlations (IFN- $\gamma$ with ITGB3, IFN- $\beta 1$ with CTSK, tartrate resistant acid phosphatase (TRAP), CALCR, RANK, RANKL, IL-1 $\alpha$ with CTSK, OPG, IL-17A with CALCR) and positive (TGF- $\beta 1$ with CTSK, TRAP, RANK), and OA specific negative (IL-1 $\alpha$ with osteoclast associated immunoglobulin-like receptor (OSCAR), TNF- $\alpha$ with RANK, RANKL, OPG) and positive (IL-6 with RANK, RANKL, OPG) correlations.

Conclusions: Our results demonstrate that the relationship between osteoclastogenic and anti-osteoclastogenic pro-inflammatory cytokines differs in human OP and OA bone and could present an important factor for characteristics of OP and OA bone phenotypes.
\end{abstract}

Keywords: Interleukins, Interferons, TNF- ${ }_{1}$, TGF- $\beta 1, \beta_{3}$ integrin, Cathepsin K, OSCAR

\footnotetext{
* Correspondence: janja.zupan@ffa.uni-lj.si

'University of Ljubljana, Faculty of Pharmacy, Department of Clinical

Biochemistry, Askerceva cesta 7, SI-1000 Ljubljana, Slovenia

Full list of author information is available at the end of the article
} 


\section{Background}

Osteoclasts are influenced by a variety of pro-inflammatory osteoclastogenic and anti-osteoclastogenic cytokines that can either stimulate or suppress their activity [1]. This regulation of osteoclasts becomes particularly important in the pathological activation of the immune system, when pro-inflammatory cytokines are produced extensively by activated T cells [2]. As the immune system is also triggered during estrogen deficiency or inflammation, both osteoporosis (OP) and osteoarthritis (OA) are being recently considered as inflammation driven bone disorders $[3,4]$. Increased levels of IL-1, TNF- $\alpha$ and IL- 6 after the menopause have been associated with OP [5-8] and higher incidence of non-traumatic fractures was associated with higher levels of serum IL-6 [9]. Furthermore, serum IL-6 accounted for up to $34 \%$ of the total variance of change in bone mineral density (BMD) after the menopause [10]. Blockade of TNF and IL-1 reduced bone resorption in postmenopausal OP women [11].

Anti-osteoclastogenic cytokines such as IFN- $\gamma$ and IFN- $\beta$ have been shown in vitro to strongly suppress osteoclastogenesis by inhibiting receptor activator of nuclear factor $\kappa \mathrm{B}$ (RANK) signalling [12,13]. However, under conditions of inflammation and estrogen deficiency, this effect of IFN- $\gamma$ could be overpowered by $\mathrm{T}$ cell secretion of RANKL and TNF- $\alpha$, resulting in net bone loss [2,14]. Transforming growth factor $\beta 1$ (TGF$\beta 1$ ) cannot be classified as a pro-inflammatory cytokine, however its role in maintaining a balance, by mediating both inhibition and stimulation of bone resorption and formation, could be deregulated by the pro-inflammatory cytokines released in pathological conditions of bone [15].

Pro-inflammatory cytokines, such as IL-1 $\alpha$, TNF- $\alpha$ and IL-17 exhibit osteoclastogenic properties [16,17] with many synergistic and also antagonizing interactions between them [18-21] and some of them, such as IL-6, may produce both stimulating and suppressing actions on osteoclasts $[17,22,23]$.

In vitro studies have shown that these cytokines can influence osteoclasts directly via their specific receptors located on osteoclasts or via modulation of the RANK/ RANK ligand (RANKL)/osteoprotegerin (OPG) system [24-26]. IL-1 $\alpha$, IL- 6 and TNF- $\alpha$ can act on osteoclasts directly or by the RANK/RANKL/OPG pathway $[16,22,27]$. Regardless of the pathway, the activation of osteoclasts leads to the expression of the osteoclast specific genes CALCR, ITGB3, OSCAR, CTSK and ACP5 that encode calcitonin receptor, $\beta_{3}$ integrin, osteoclast associated immunoglobulin-like receptor (OSCAR), cathepsin $\mathrm{K}$ and tartrate resistant acid phosphatase (TRAP) respectively, all of which are involved in differentiation, activation and survival of osteoclasts [28].
These data, obtained mainly from in vitro studies and experimental animal models, indicate complex crosstalk between the pro-inflammatory cytokines, and strongly suggest that the exact outcome of the specific cytokine must be evaluated in pathological conditions of the bone microenvironment. One of the first studies on human bone biopsies, performed by Ralston in 1994, demonstrated that the expression of IL- $1 \alpha$, IL- $1 \beta$, TNF$\alpha$ and IL- 6 is more frequent in women with OP fractures than in normal postmenopausal women [29]. Recently, D'Amelio et al. found up regulation of expression of RANKL and OPG genes in OP, while TGF- $\beta$ was highly expressed in OA women [30]. To the best of our knowledge the expression of anti-osteoclastogenic cytokines, such as IFN- $\gamma$ and IFN- $\beta$, has not been quantified in human OP or OA bone tissue so far. Due to ethical reasons, normal human bone tissue is difficult to obtain and studies utilizing normal human iliac crest bone [29] and intertrochanteric bone obtained from cadavers at autopsy $[31,32]$ are limited.

$\mathrm{OP}$ and $\mathrm{OA}$ are two contrasting bone phenotypes in terms of BMD [33], and both have only recently been considered as inflammatory bone disorders $[3,4]$ in which osteoclastogenic and anti-osteoclastogenic cytokines might play important roles.

Given these observations the aim of our study was to investigate, whether there is a difference between OP and OA: first, in the expression of the osteoclastogenic and anti-osteoclastogenic cytokines, second, in correlation of these cytokines with BMD and levels of bone turnover markers (BTM) and third, in correlation of these cytokines with osteoclast specific genes and RANK/RANKL/OPG genes.

Therefore, the gene expression of 15 pro-inflammatory cytokines including their receptors, TGF- $\beta 1$ and its receptor, 3 genes of the RANK/RANKL/OPG system and 5 osteoclast specific genes, in human OP and OA bone tissue, was examined. IFN- $\gamma$ and IFN- $\beta$ have been quantified for the first time in human OP and OA bone tissue.

\section{Methods}

\section{Human bone tissue samples}

Gene expression profiles were determined in bone samples from fifty four (54) patients undergoing hemiarthroplasty or total hip arthroplasty because of lowenergy femoral neck fracture (23 OP patients) or primary hip OA (31 OA patients). Patients were included in the study in a consecutive manner over a period of 1.5 years as they were directed to arthroplasty at the Department of Traumatology in the General Hospital Celje because of diagnosis of OP or OA. OP was diagnosed by radiologically confirmed low-energy femoral 
neck fracture and diagnosis of OA was established by clinical and radiographic criteria according to the Harris hip score [34]. All OP patients were submitted to arthroplasty within $24 \mathrm{~h}$ following femoral neck fracture. Bone tissue samples (approximately $1 \mathrm{~cm}^{3}$ ) were collected during surgical procedures of femoral osteotomy from the trabecular bone at the metaphyseal cutting plane. Bone samples were immediately frozen in liquid nitrogen and stored at $-80^{\circ} \mathrm{C}$ until RNA extraction. The exclusion criteria for enrollment of OP and OA patients, verified by the questionnaire, laboratory results and interview, included the following: secondary OP or OA, liver and kidney diseases, endocrinological disorders and medical anamnesis on receiving medications with known influence on bone metabolism. The study was approved by the ethical committee of Republic of Slovenia and all patients gave written informed consent.

\section{Bone mineral density measurement}

BMD at the contralateral hip, lumbar spine (L2-L4) and femoral neck was measured by dual-energy X-ray absorptiometry (Hologic QDR 1000, Hologic, Inc. Bedford). The measurement of BMD in OA patients was performed pre-operatively and in OP patients immediately post-operatively.

\section{Biochemical markers of bone turnover}

Biochemical BTM were measured in a subset of 28 patients (12 OP and 16 OA) within 24 days after surgery. Blood samples were collected between 8:00 a.m. and 10:00 a.m. after an overnight fast. Serum C-terminal crosslinking telopeptides of type I collagen (CTX), serum free soluble RANKL, cathepsin K and OPG were measured by enzyme immunoassay (Serum CrossLaps ELISA, Nordic Bioscience Diagnostics A/S, Herlev, Denmark; sRANKL ELISA, Cathepsin K ELISA and Osteoprotegerin ELISA, Biomedica, Vienna, Austria) with an inter-assay coefficient of variation (CV) below $10 \%, 6 \%$, $8 \%$ and $8 \%$, respectively. Osteocalcin (OC) in heparinized plasma was measured by a solidphase, two-site chemiluminescent enzyme-labelled immunometric assay (Immulite Osteocalcin, Diagnostic Product Corporation, Los Angeles, CA, USA).

\section{Quantitative real-time polymerase chain reaction}

Total RNA was extracted from human bone samples and complementary DNA (cDNA) synthesized according to our previously described procedure [35] and stored at $-80^{\circ} \mathrm{C}$ until measurement of gene expression.

Predesigned and validated gene-specific TaqMan Gene Expression Assays (Applied Biosystems, Foster, CA, USA) for RANK (TNFRSF11A), IFN- $\gamma$ (IFNG), IL1A, IL$1 \alpha$ receptor type I (IL1R1), IL-6 (IL6), TNF- $\alpha$ receptor (TNFRSF1A) and OSCAR genes (Hs00921374_m1,
Hs99999041_m1, Hs00899848_m1, Hs00168392_m1, Hs00174131_m1, Hs01042313_m1 and Hs01100185, respectively) were used according to the manufacturer's protocol. For the remaining genes, oligonucleotides (Sigma Aldrich Chemie Gmbh, Munich, Germany) for mRNA encoding IFN- $\gamma$ receptor (IFNGR1), IFN- $\beta$ (IFNB1), both chains of IFN $-\beta$ receptor (IFNAR1 and IFNAR2), IL-1 $\alpha$ receptor type II (IL1R2), IL-6 receptor (IL6R), IL-17A (IL17A), two IL-17A receptors A and C (IL17RA and IL17RC), TNF- $\alpha$ (TNF), TGF- $\beta 1$ (TGFB1), TGF- $\beta 1$ receptor T $\beta$ R1 (TGFBR1), calcitonin receptor $(C A L C R)$ and $\beta_{3}$ integrin (ITGB3) were designed using Primer-BLAST (NCBI). Real time quantification was carried out on LightCycler 480 (Roche Diagnostics Ltd, Rotkreuz, Switzerland) using $5 \times$ HOT FIREPol EvaGreen qPCR Mix Plus for oligonucleotides and 5× HOT FIREPol Probe qPCR Mix Plus (Solis BioDyne, Tartu, Estonia) for TaqMan assays. All samples were quantified in triplicate. Dilution series of cDNA were prepared to create a relative standard curve with each run and absolute quantification of the data was performed using the second derivative maximum method (LightCycler 480, Software Version 1.5, Roche Diagnostics Ltd, Rotkreuz, Switzerland). All data were normalized to the geometric mean of two internal housekeeping genes, glyceraldehyde-3-phosphate dehydrogenase (GAPDH) and ribosomal protein, large, P0 (RPLPO). Data on TNFSF11, TNFRSF11B, ACP5 and CTSK expression were taken from our previous study on the same group of patients [35] and the appropriate correction factor, according to expression of two housekeeping genes, was applied.

\section{Statistical analysis}

Variables were tested for normality of distribution using the Shaphiro-Wilk test. For variables such as age, sex, body mass index and BMD, that have met the normality assumption, Student's $t$-test was used for comparison between the two groups of patients. Because of the nonparametric distribution of the BTM levels and mRNA data, Mann-Whitney $U$ test was used for comparison between the two tissue groups, and Spearman rho correlation analysis for estimating the relationship between the gene expression data, BMD and BTM, and within the mRNA data, respectively. Results with a $p$ value of 0.05 or less were considered statistically significant. All data analyses were performed using PASW software, version 18 (IBM, Chicago, IL, USA).

\section{Results}

\section{Study population}

The study population consisted of age and sex matched patients with femoral neck fracture due to low-energy trauma (OP) and patients with arthroplastic surgery of the hip (OA). The two groups differed in body mass 
index (BMI) and BMD values of the hip, femoral neck and lumbar spine, these values being significantly lower in OP than in OA. Cathepsin K and OPG serum levels were significantly higher in OP (Table 1 ).

\section{Gene expression measurement}

The expression of 25 genes encoding 15 pro-inflammatory cytokines and their receptors (IL1A, IL6, IFNG, IFNB1, IL17A, TNF, IL1R1, IL1R2, IL6R, IFNG1, IFNAR1, IFNAR2, IL17RA, IL17RC, TNFRSF1A), TGF$\beta 1$ and its receptor (TGFB, TGFBR1), 3 genes of the RANK/RANKL/OPG system (TNFRSF11A, TNFSF11, TNFRSF11B) and 5 osteoclast specific genes (ITGB3, OSCAR, CTSK, ACP5, CALCR) in human OP and OA bone tissue was measured. All mRNA values were normalized to the geometric mean of GAPDH and RPLPO mRNA. Ligand to receptor mRNA levels for each of the studied cytokine ligand receptor pairs were calculated from normalized expression data and these ratios were used further in our correlation analyses. Excluding Background, the name of the specific pro-inflammatory cytokine is referred to the corresponding ligand receptor mRNA ratio throughout the manuscript.

\section{Difference in gene expression between OP and OA}

The results of Mann-Whitney U tests showed no differences between males and females in the whole study group or within OP or OA groups in the expression levels of genes measured.

The expression of osteoclastogenic and of antiosteoclastogenic cytokines between $\mathrm{OP}$ and $\mathrm{OA}$ tissues were compared using the Mann-Whitney $U$ test. In

Table 1 Anthropometric characteristics and serum levels of bone turnover markers

\begin{tabular}{lll}
\hline & Osteoporosis & Osteoarthritis \\
\hline Age (years) & $74.1 \pm 7.7$ & $71.8 \pm 4.2$ \\
\hline Sex (women/men) & $17 / 6$ & $23 / 8$ \\
\hline Body mass index $\left(\mathbf{k g} / \mathbf{m}^{2}\right)$ & $24.5 \pm 2.3$ & $28.3 \pm 4.4^{* *}$ \\
\hline Hip bone mineral density $\left(\mathbf{g} / \mathbf{c m}^{2}\right)$ & $0.726 \pm 0.135$ & $0.880 \pm 0.140^{* *}$ \\
\hline Femoral neck BMD $\left(\mathbf{g} / \mathbf{c m}^{2}\right)$ & $0.615 \pm 0.098$ & $0.757 \pm 0.131^{* *}$ \\
\hline Lumbar spine L2-L4 BMD $\left(\mathbf{g} / \mathbf{c m}^{2}\right)$ & $0.861 \pm 0.184$ & $0.994 \pm 0.193^{*}$ \\
\hline CTX (pmol/l) & $3078(1285 ; 5391)$ & $3547(2183 ; 4967)$ \\
\hline Osteocalcin $(\boldsymbol{\mu g} / \mathbf{l})$ & $6.80(0.30 ; 11.98)$ & $7.90(1.03 ; 17.03)$ \\
\hline Cathepsin K (pmol/l) & $12.0(10.0 ; 22.1)$ & $9.4(6.7 ; 11.3)^{*}$ \\
\hline RANKL (pmol/l) & $0.01(0.01 ; 0.02)$ & $0.01(0.01 ; 0.06)$ \\
\hline OPG (pmol/l) & $5.86(4.52 ; 7.47)$ & $4.42(4.09 ; 5.01)^{*}$ \\
\hline
\end{tabular}

$B M D$ bone mineral density. CTX C-terminal crosslinking telopeptides of type I collagen. RANKL receptor activator of nuclear factor $\kappa \mathrm{B}$ ligand. $O P G$ osteoprotegerin.

Values are means \pm standard deviation (with the exception of sex) and medians(25th;75th quartile) for bone turnover markers.

Comparisons were assessed with Student's t-test or Mann-Whitney $U$ test, ${ }^{*} p$ $<0.05,{ }^{* *} p<0.001$.
OP tissue, a significantly higher expression of ligand to receptor mRNA levels for IL1A/IL1R1, IL1A/IL1R2 and $I L 6 / I L 6 R$ were observed, while in OA tissue, there was a significantly higher expression of IFN- $\gamma$ ligand to receptor mRNA (Figure 1). Looking at the osteoclast specific genes, there was higher expression of OSCAR and CALCR in OA, while higher expression of $A C P 5$ in OP was of limited statistical significance ( $p=$ 0.049). TNFRSF11A and TNFSF11 were both significantly higher in OP, while their ratio did not reach the level of significance. TNFRSF11B was similar between both groups, while the ratio with RANKL (TNFSF11/TNFRSFS11B) was significantly higher in OP (Table 2).

\section{Correlation of the pro-inflammatory cytokines with bone mineral density}

The correlation between cytokine ligand to receptor mRNA levels and BMD was determined by nonparametric analysis. In OP, significant negative association with both, hip and femoral neck BMD was found for RANKL/RANK (rho $=-0.452$ and $-0.443, p<0.05$ ). In $\mathrm{OA}$, significant negative correlation was observed for TNF/TNFRSF1A with hip BMD (rho $=-0.390, p<0.05$ ), while correlation with femoral neck BMD did not reach the level of significance (rho $=-0.355, p=0.058$ ).

Significant negative correlations of RANKL/OPG with total hip and femoral neck BMD $(p=-0.306$ and -0.383 , $p<0.05)$ were found in the whole study group only.

\section{Correlation of the pro-inflammatory cytokines with bone turnover markers}

The correlation between cytokine ligand to receptor mRNA levels and BTM was determined by nonparametric analysis. In OP, significant positive association for IL1A/IL1R2 with serum RANKL and negative for IFN- $\gamma$ ratio with serum cathepsin $\mathrm{K}$ have been found (rho $=0.608$ and $-0.634, p<0.05$ ). In OA, significant positive correlation was observed for TGF- $\beta 1$ ratio with serum OPG and TNF- $\alpha$ ratio with cathepsin $\mathrm{K}$ (rho = 0.636 and $0.591, p<0.05$ ), and a negative correlation of IL-6 ratio with cathepsin $\mathrm{K}$ (rho $=-0.521, p<0.05)$. Correlations between RANKL, RANKL/RANK, RANKL/ OPG mRNA and BTM were not significant for any of the groups studied $(p>0.05)$, while OPG mRNA showed significant negative correlation with serum OPG in the OA group (rho $=-0.518, p<0.05$ ).

\section{Correlation of the pro-inflammatory cytokines with osteoclast specific and RANK/RANKL/OPG genes}

The correlation between cytokine ligand to receptor mRNA levels and osteoclast specific genes, RANK, RANKL and OPG gene mRNA was examined by nonparametric analysis (Table 3). 


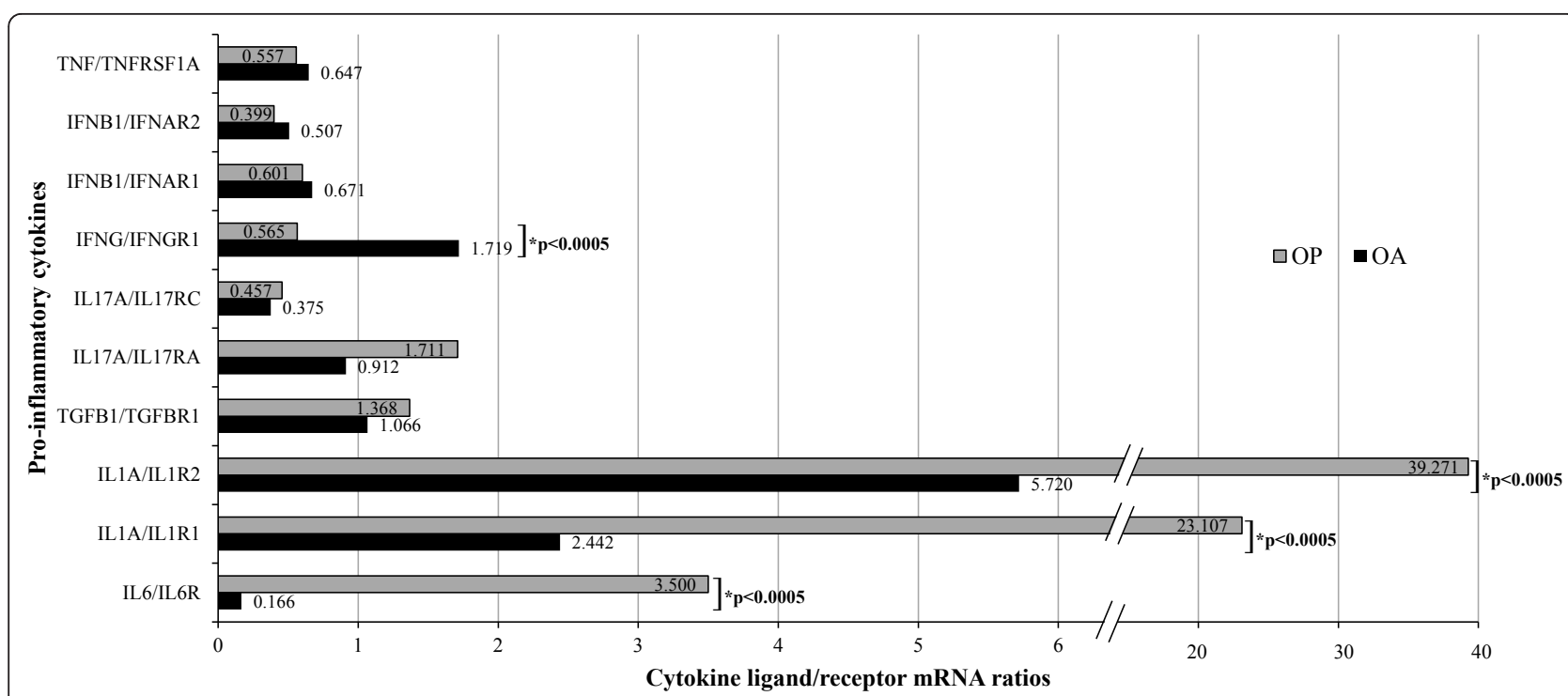

Figure 1 Quantitative real-time PCR data of the pro-inflammatory cytokine mRNA levels in osteoporosis (OP) and osteoarthritis (OA) Ligand to receptor ratios for each of the studied cytokine ligand receptor pairs were calculated from mRNA values normalized to geometric mean of GAPDH and RPLPO mRNA. Values are medians. Comparisons were assessed by the Mann-Whitney $U$ test, ${ }^{*} p$ values $<0.05$ were considered statistically significant.

In OP specifically, IL-6 showed a significant positive correlation with ITGB3 and TGF- $\beta 1$ with CTSK and $A C P 5$. Significant negative correlations were found for the IL-6 with CTSK and CALCR, IL1A/IL1R1 ratio with $C T S K$, and IL-17A with CALCR for ligand to receptor ratios with both of its receptors IL-17RA and IL-17RC, while IL17A/IL17RA also showed negative correlation with CTSK. Significant negative correlation of both IL1A/IL1R1 and IL1A/IL1R2 with OPG was found. Only TGF- $\beta 1$ showed significant positive correlation with RANK. Negative correlations were demonstrated for IL-
17A ligand to receptor ratios, with both IL-17RA and IL-17RC, and for IL-6 ratio with RANK.

Anti-osteoclastogenic cytokines in OP tissue showed a significant negative correlation for IFN- $\gamma$ ligand to receptor ratio (IFNG/IFNGR1) with ITGB3 and for IFN- $\beta$ ratio with CTSK, CALCR and ACP5. The latter observations were found for both ligand to receptor mRNA ratios, IFNB1/IFNAR1 and IFNB1/IFNAR2, as there are two protein chains of IFN- $\beta$ receptor. A significant negative correlation of both IFNB1/IFNAR1 and IFNB1/IFNAR2 with RANK and RANKL gene expression was also found.

Table 2 Data on mRNA levels of osteoclast specific genes and RANK/RANKL/OPG genes in OP and OA

\begin{tabular}{|c|c|c|c|c|c|}
\hline Gene symbol & Gene name & $\mathrm{OP}(\mathrm{n}=23)$ & $O A(n=31)$ & $O P / O A^{a}$ & $p$ value $^{\mathrm{b}}$ \\
\hline \multicolumn{6}{|c|}{ Osteoclast specific genes } \\
\hline OSCAR & Osteoclast associated immunoglobulin-like receptor & $0.574(0.345 ; 0.854)$ & $1.002(0.732 ; 1.339)$ & 0.54 & $0.002^{*}$ \\
\hline ITGB3 & $\beta_{3}$ integrin & $0.241(0.074 ; 0.586)$ & $0.285(0.143 ; 0.994)$ & 0.85 & 0.231 \\
\hline CTSK & Cathepsin K & $0.288(0.121 ; 1.109)$ & $0.263(0.136 ; 0.555)$ & 1.10 & 0.786 \\
\hline ACP5 & Tartrate resistant acid phosphatase (TRAP) & $0.270(.128 ; 1.011)$ & $0.147(0.073 ; 0.259)$ & 1.84 & $0.049 *$ \\
\hline CALCR & Calcitonin receptor & $0.223(0.138 ; 0.644)$ & $0.860(0.482 ; 1.562)$ & 0.26 & $<0.0005^{* *}$ \\
\hline \multicolumn{6}{|c|}{ RANK/RANKL/OPG genes } \\
\hline TNFRSF11A & Receptor activator of nuclear factor $\kappa$ B (RANK) & $1.580(0.687 ; 3.939)$ & $0.573(0.355 ; 1.038)$ & 2.76 & $0.002^{*}$ \\
\hline TNFSF11 & Receptor activator of nuclear factor $\kappa \mathrm{B}$ ligand (RANKL) & $0.528(0.076 ; 1.038)$ & $0.091(0.027 ; 0.253)$ & 5.80 & $<0.0005^{* *}$ \\
\hline TNFRSF11B & Osteoprotegerin (OPG) & $0.699(0.410 ; 0.914)$ & $0.776(0.308 ; 1.615)$ & 0.90 & 0.681 \\
\hline TNFSF11/TNFRSF11A & RANKL/RANK & $0.241(0.083 ; 0.928)$ & $0.140(0.064 ; 0.271)$ & 1.72 & 0.076 \\
\hline TNFSF11/TNFRSF11B & RANKL/OPG & $0.448(0.295 ; 1.601)$ & $0.112(0.075 ; 0.193)$ & 4.00 & $<0.0005^{* *}$ \\
\hline
\end{tabular}

Values are medians(25th;75th percentile). All mRNA values were normalized to the geometric mean of GAPDH and RPLPO mRNA.

${ }^{a}$ Gene expression ratio of medians in osteoporotic (OP) versus osteoarthritic (OA) patients.

${ }^{\mathrm{b}}$ Comparisons were assessed by the Mann-Whitney $U$ test. ${ }^{*} p<0.05$, ${ }^{* *} p<0.001$. 
Table 3 Correlation of the expression of the pro-inflammatory cytokines and osteoclasts' specific genes and RANK/RANKL/OPG genes

\begin{tabular}{|c|c|c|c|c|c|c|c|c|c|c|c|c|c|c|c|c|}
\hline \multirow{2}{*}{$\begin{array}{l}\text { Gene mRNA } \\
\text { Ligand/Receptor mRNA ratio }\end{array}$} & \multicolumn{2}{|c|}{ OSCAR } & \multicolumn{2}{|c|}{$\beta 3$ integrin } & \multicolumn{2}{|c|}{ Cathepsin K } & \multicolumn{2}{|c|}{ TRAP } & \multicolumn{2}{|c|}{ Calcitonin receptor } & \multicolumn{2}{|c|}{ RANK } & \multicolumn{2}{|c|}{ RANKL } & \multicolumn{2}{|c|}{ OPG } \\
\hline & OP & $O A$ & OP & $O A$ & OP & $O A$ & OP & $O A$ & OP & $O A$ & OP & $O A$ & OP & $O A$ & OP & $O A$ \\
\hline IL6/IL6R & -0.282 & -0.215 & $0.587^{*}$ & $-0.363^{*}$ & $-0.451^{*}$ & $\underline{0.519 * *}$ & -0.329 & 0.319 & $-0.431^{*}$ & $\underline{0.656^{* *}}$ & -0.421 & $0.658^{* *}$ & 0.083 & $\underline{0.619 * *}$ & -0.147 & $0.529 * *$ \\
\hline IL1A/IL1R1 & -0.181 & -0.156 & 0.239 & 0.137 & $-0.508^{*}$ & -0.166 & -0.350 & 0.021 & $-0.494^{*}$ & $-0.434^{*}$ & -0.311 & -0.301 & -0.259 & -0.258 & $-0.484^{*}$ & -0.296 \\
\hline IL1A/IL1R2 & -0.021 & $-0.380^{*}$ & 0.100 & -0.005 & -0.103 & 0.325 & 0.015 & 0.348 & -0.084 & 0.207 & 0.027 & 0.305 & -0.066 & 0.337 & $-0.491^{*}$ & 0.271 \\
\hline TGFB1/TGFBR1 & 0.054 & -0.198 & 0.112 & 0.256 & $\underline{0.473^{*}}$ & -0.075 & $\underline{0.486^{*}}$ & 0.048 & 0.302 & -0.201 & $\underline{0.531^{*}}$ & -0.051 & 0.263 & -0.208 & 0.027 & -0.033 \\
\hline IL17A/IL17RA & 0.007 & 0.030 & -0.116 & 0.167 & $-0.491^{*}$ & -0.081 & -0.263 & -0.084 & $-0.600^{* *}$ & 0.087 & $-0.605^{* *}$ & 0.002 & -0.293 & -0.009 & 0.068 & 0.021 \\
\hline IL17A/IL17RC & -0.140 & 0.010 & -0.358 & -0.070 & -0.390 & -0.154 & 0.213 & -0.004 & $-0.600^{*}$ & -0.095 & $-0.537^{*}$ & -0.096 & -0.005 & -0.027 & 0.213 & -0.004 \\
\hline IFNG/IFNGR1 & 0.198 & -0.181 & $-0.601^{* *}$ & -0.062 & 0.119 & 0.205 & -0.018 & 0.097 & 0.191 & 0.120 & -0.070 & 0.207 & -0.197 & 0.296 & 0.337 & 0.167 \\
\hline IFNB1/IFNAR1 & 0.224 & 0.006 & 0.095 & 0.188 & $-0.553^{* *}$ & -0.283 & $-0.453^{*}$ & -0.290 & $-0.527^{* *}$ & -0.038 & $-0.580^{* *}$ & -0.202 & $-0.447^{*}$ & -0.186 & 0.002 & -0.070 \\
\hline IFNB1/IFNAR2 & 0.313 & -0.049 & 0.013 & 0.107 & $-0.510^{*}$ & -0.137 & $-0.430^{*}$ & -0.152 & $-0.463^{*}$ & 0.064 & $-0.517^{*}$ & -0.052 & $-0.456^{*}$ & -0.035 & 0.043 & 0.063 \\
\hline TNF/TNFRSF1A & 0.329 & 0.292 & -0.038 & 0.018 & $-0.428^{*}$ & $-0.632^{* *}$ & $-0.429^{*}$ & $-0.598^{* *}$ & $-0.414^{*}$ & $-0.613^{* *}$ & -0.362 & $-0.631^{* *}$ & -0.356 & $-0.541^{* *}$ & 0.192 & $-0.534^{* *}$ \\
\hline
\end{tabular}

OSCAR osteoclast associated immunoglobulin-like receptor. TRAP tartrate resistant acid phosphatase. RANK receptor activator of nuclear factor $\kappa B$. RANKL RANK ligand. OPG osteoprotegerin.

The numbers are Spearman's rho coefficients for osteoporotic (OP) and osteoarthritic (OA) tissue. $p$ values were obtained using nonparametric bivariate correlation analysis, ${ }^{*} p<0.05,{ }^{* *} p<0.01$. Significant positive correlations are underlined with bold line. 
In OA tissue, interestingly, correlations directly opposite to those in OP tissue were found for IL-6 ratio with ITGB3, CTSK and CALCR. Furthermore, the ratio of IL$1 \alpha$ to its receptor type II (IL1A/IL1R2) showed a negative correlation with OSCAR. Strong negative correlation of TNF- $\alpha$ ratio with RANK, RANKL and OPG, and an opposite, positive correlation of IL-6 ratio with the same genes, were found.

No significant correlations for IFN- $\gamma$ and IFN $-\beta$ were found with any of the osteoclast specific genes in OA.

In both tissues, IL1A/IL1R1 was significantly inversely correlated with $C A L C R$, while TNF- $\alpha$ ratio showed significant negative correlations with CTSK, ACP5 and CALCR.

\section{Discussion}

We have demonstrated that the relationship between osteoclastogenic and anti-osteoclastogenic pro-inflammatory cytokines differs in human OP and OA bone.

The comparison of OP and OA showed higher expression of osteoclastogenic cytokines IL- 6 and IL- $1 \alpha$ in OP and higher expression of IFN- $\gamma$ in OA. Negative association with BMD has been found for RANKL in OP and for TNF- $\alpha$ in OA. In OP, positive association of IL- $1 \alpha$ with serum RANKL and negative of IFN- $\gamma$ with serum cathepsin $\mathrm{K}$ have been found, while in OA, positive correlation of TGF- $\beta 1$ with serum OPG and of TNF- $\alpha$ with cathepsin $\mathrm{K}$, and negative correlation of IL- 6 with cathepsin K were observed. The correlations of IL-6 with osteoclasts specific genes ITGB3, CTSK and CALCR expression were directly opposite between $\mathrm{OP}$ and $\mathrm{OA}$. The negative correlations specific to OP were IL- $1 \alpha$ with CTSK and OPG, IL-17A with CALCR, IFN- $\gamma$ with ITGB3, IFN- $\beta$ with CTSK, ACP5, CALCR, RANK and RANKL and positive correlations specific to OP were TGF- $\beta 1$ with RANK, TRAP and cathepsin $\mathrm{K}$. The negative correlations specific for OA were IL- $1 \alpha$ with OSCAR and TNF- $\alpha$ with RANK, RANKL, OPG, and positive OA specific IL-6 with RANK, RANKL and OPG.

$\mathrm{OP}$ and $\mathrm{OA}$ are both age related skeletal disorders, in which the involvement of pro-inflammatory cytokines, due to pathological activation of the immune system, was recently suggested $[3,4]$. As OP and OA are two opposite bone phenotypes in terms of BMD [33], our hypothesis was that the two types of tissue differ in the relationship between osteoclastogenic and anti-osteoclastogenic pro-inflammatory cytokines and in the correlation of these pro-inflammatory cytokines with BMD and BTM. Bone samples were collected from OP and OA patients with significantly different BMD at three skeletal sites (Table 1). The results show higher expression of IL- 6 and IL- $1 \alpha$ in OP, while the expression of IFN- $\gamma$ was higher in OA tissue. Although our results were significant only for IL-1 $\alpha$, IL- 6 and IFN- $\gamma$, higher expression of all osteoclastogenic cytokines studied, except TNF- $\alpha$ was implied in OP, and higher expression of the anti-osteoclastogenic cytokines IFN- $\gamma$ and IFN- $\beta$ in OA (Figure 1). The higher expression of genes encoding bone resorption molecules TRAP, RANK and RANKL and RANKL/OPG in OP tissue confirms the adequacy of the system used in our study. In OA, $O S C A R$ and $C A L C R$ were significantly higher expressed (Table 2). Interestingly, a previous study comparing OA with controls from cadavers found no differences in CALCR expression [32].

Our results show that the relationship between osteoclastogenic cytokines IL- 6 and IL- $1 \alpha$ and the anti-osteoclastogenic cytokine IFN- $\gamma$ is diametrically opposite in $\mathrm{OP}$ and $\mathrm{OA}$, corresponding to their bone phenotype. In accordance with in vitro studies, higher level of the antiosteoclastogenic cytokine IFN- $\gamma$ in OA might contribute to the suppressed osteoclast activity [13], while higher levels of the osteoclastogenic cytokines IL- 6 and IL-1 $\alpha$ in OP could suggest enhanced osteoclastogenesis, leading to increased bone loss $[1,16,20,22]$. Our results of higher expression of IL- 6 and IL- $1 \alpha$ in OP and no difference in TNF- $\alpha$ expression are in accordance with those of Ralston [29]. Furthermore, our results for IL-6 also coincide with those of Cauley et al. who showed that high serum levels of IL-6 predict a higher incidence of non-traumatic fractures. They found similar association for TNF- $\alpha$, while in our study TNF- $\alpha$ ratio showed negative correlation with total hip BMD in OA [9]. Nevertheless, the negative correlations of RANKL/ RANK in OP and RANKL/OPG in the whole group with total hip and femoral neck BMD found in our study are in accordance with the well-established role of the RANK/RANKL system in bone [24]. We have found tissue specific correlation of the pro-inflammatory cytokines gene expression and serum levels of BTM such as cathepsin K, RANKL and OPG. In OP, positive association of IL-1 $\alpha$ with serum RANKL might indicate the osteoclastogenic activity of IL- $1 \alpha$, while negative association of IFN- $\gamma$ with serum cathepsin $\mathrm{K}$ might indicate anti-osteoclastogenic activity of IFN- $\gamma$, previously confirmed in vitro in mice [36]. In OA, positive correlation for TGF- $\beta 1$ with serum OPG and a negative correlation of IL-6 with cathepsin K, that could indicate the antiosteoclastogenic actions of these cytokines, resulting in bone sparing effect in OA, were found. The latter observation for IL-6 is in accordance with the previously observed dual role of IL- 6 on osteoclasts $[17,22,23]$. Although previous reports have shown an inverse relationship between RANKL bone mRNA and serum levels in OA males [37], our results of this correlation analysis did not reach the level of significance. The reason is most likely due to low statistical power as there were 
only three OA males with serum RANKL levels. Interestingly, we observed significant negative correlation between OPG mRNA and serum OPG in the OA group although Findlay et al. have found the opposite correlation.

Our results of non-significant higher expression of TGF- $\beta 1$ ligand gene TGFB 1 in OA $(p=0.053$, data not shown) and higher expression of RANKL/OPG in OP ( $p$ $<0.0005)$ correspond to results of the recent D'Amelio et al. study, that they have also verified on the protein level [30]. To the best of our knowledge, the expression of IFN $-\gamma$ and IFN $-\beta$ has not been previously quantified in human OP or OA bone tissue.

Moreover, we performed a correlation analysis of the pro-inflammatory cytokines and TGF- $\beta 1$ expression with osteoclast specific and RANK/RANKL/OPG genes. Again, the differences between OP and OA were found. IL-6 showed positive correlation with $\beta_{3}$ integrin expression in OP, while a negative link with the same gene has been found in OA. Furthermore, negative correlation of IL-6 expression with genes encoding RANK, cathepsin $\mathrm{K}$ and calcitonin receptor in OP and the positive correlation with the same genes in $\mathrm{OA}$, have been found. The results for IL-6 in OA are in accordance with the previously observed opposing data on IL- 6 activity on osteoclasts $[17,22,23]$. Nevertheless, the 21 -fold higher expression of IL- 6 in OP and its positive correlation with $\beta_{3}$ integrin expression could indicate its osteoclastogenic effect in OP. Furthermore, both IL- 6 and IFN- $\gamma$ have shown directly opposite correlation with $\beta_{3}$ integrin expression between $\mathrm{OP}$ and $\mathrm{OA}$, and also reverse correlation between them (rho $=-0.463, p<0.05$, data not shown) that was specific for OP.

Another osteoclastogenic cytokine in our study, IL-1 $\alpha$ has shown positive correlation with serum RANKL and negative correlation with OPG gene expression in OP that might indicate its osteoclastogenic pathway. The negative association of IL- $1 \alpha$ with cathepsin K gene expression is contrary to the results of Kamolmatyakul et al. in mice. Negative correlations of IL- $1 \alpha$ with OSCAR gene expression specific to OA could indicate the dual role of IL- $1 \alpha$, similar to that of IL- 6 . Nevertheless, IL-1 $\alpha$ showed almost 10 -fold higher expression in OP than in OA. Our results might suggest that IL-1 $\alpha$ in OP could act via suppression of the OPG, the decoy receptor for RANKL, resulting in less inhibition of RANKL, increased serum RANKL and enhanced osteoclast activity observed in OP. Additional studies are needed to confirm this suggestion.

The differences between OP and OA were also found for TGF- $\beta 1$, a ubiquitous growth factor retaining a balance in coupling bone resorption and formation [15]. TGF- $\beta 1$ showed positive correlation with cathepsin K, TRAP and RANK gene expression in OP that could suggests the involvement of TGF- $\beta 1$ in enhanced osteoclastogenesis in OP. Similarly, Yan et al. showed that TGF- $\beta 1$ stimulates the expression of $C A L C R$ and RANK in TRAP positive osteoclasts in isolated murine monocytic cells [38]. On the other hand, TGF- $\beta 1$ positive correlation with serum OPG in OA indicates its opposite, anti-osteoclastogenic effect in OA. Furthermore, our results showed a negative correlation of TGF- $\beta 1$ with IFN $-\beta$ in OP tissue (rho $=-0.536, p<0.05$, data not shown), that is in accordance with the mechanism proposed by Lovibond et al., in which TGF- $\beta 1$ stimulates osteoclast formation via suppression of the anti-osteoclastogenic effect of IFN- $\beta$ [19].

Looking at the anti-osteoclastogenic cytokines in our study, OP specific negative correlations of IFN $-\gamma$ with $\beta_{3}$ integrin and of IFN $-\beta$ with genes encoding cathepsin $K$, calcitonin receptor, TRAP, RANK and RANKL were found, that are in accordance with the anti-osteoclastogenic effect of IFN- $\gamma$ and IFN- $\beta$, proved in vitro $[12,13]$. These relationships were specific for OP, although we would have expected such connections indicating the bone sparing effect in OA. However, in our study, IFN- $\gamma$ was 3-fold higher expressed in OA associated with high bone mass. Previously, IFN- $\gamma$ has been shown to down regulate cathepsin $\mathrm{K}$ expression in co-cultures of mouse osteoclasts and stromal cells [36], while in our study, negative correlation of IFN $-\gamma$ with $\beta_{3}$ integrin gene was found that has not been described before and should be further examined. Taking together the results for IL-6 and IFN- $\gamma$, i.e. their inverse relationship, their inverse link to their common target $\beta_{3}$ integrin gene, and their inverse expression between OP and OA, we suggest that the difference in relationship between osteoclastogenic cytokine IL- 6 and anti-osteoclastogenic cytokine IFN- $\gamma$ could contribute to different osteoclast activity and thus might present an important factor for the distinctive bone phenotypes seen in OP and OA.

For the last two cytokines TNF- $\alpha$ and IL-17A no difference between OP and OA was observed, moreover the negative correlations with osteoclast specific and RANK/RANKL/OPG genes are contrary to the previous reports on their osteoclastogenic activity $[16,17,21,25]$. Negative association of TNF- $\alpha$ with BMD, RANK, RANKL and OPG gene expression and a positive with serum cathepsin $\mathrm{K}($ rho $=0.591, p<0.05)$, might also indicate the dual role of TNF- $\alpha$ on osteoclastogenesis. However, further studies are needed.

To summarize, our results of 1) higher expression of IL- 6 and IL- $1 \alpha$ in OP, OP specific negative correlation of RANKL with BMD, positive of IL- $1 \alpha$ with serum RANKL and negative correlation of IFN- $\gamma$ with serum cathepsin $K$, and a positive link of IL- 6 with $\beta_{3}$ integrin expression and negative of IL- $1 \alpha$ with OPG 2) TGF- $\beta 1$ positive correlation with cathepsin K, TRAP and RANK 
gene expression in OP and TGF- $\beta 1$ positive correlation with serum OPG in OA and 3) higher expression of IFN- $\gamma$ in OA, IFN- $\gamma$ negative link to $\beta_{3}$ integrin expression, and IFN $-\beta$ negative correlation with cathepsin $\mathrm{K}$, calcitonin receptor, TRAP, RANK and RANKL gene expression in OP, demonstrate the difference in the relationship between osteoclastogenic and anti-osteoclastogenic pro-inflammatory cytokines in OP and OA bone.

The limitations of the current study present a relatively low number of patients with BTM and the lack of further functional confirmation of the significant associations found in our study. As blood samples in this study were collected after surgery, it might be argued there could have been influence of surgery or immobilization on levels of BTM. To test this hypothesis, initial comparison of BTM levels in blood samples collected within 7 days after surgery with blood samples collected within 8-24 days after surgery was performed, and the results showed no influence of the time of blood specimen collection on the levels of BTM studied. Due to difficulty in obtaining normal human bone tissue, no controls such as cadaveric bone samples [31,32], were included in our study. However, the augmentation of the pro-inflammatory cytokines is characteristic of the pathological conditions of bone, such as inflammatory, age and hormone related bone disorders. The advantage of our study is that we evaluated the human bone tissue ex vivo as it is by comparing the steady-state of the proinflammatory cytokine gene expression in bone tissue, originating from bone and bone marrow cells, between the two opposite bone phenotypes. To avoid possible post-fracture response affecting the steady-state of gene expression, samples were taken from intertrochanteric region, located distal to the site of fracture. The significant correlations found in our study suggest the pathways for further evaluation of the pro-inflammatory cytokines in OP and OA.

\section{Conclusions}

In conclusion, we have found that the relationship between osteoclastogenic and anti-osteoclastogenic proinflammatory cytokines differs in human OP and OA bone tissue, corresponding to their specific bone phenotypes. Our data support the recent reports on the role of inflammation in OP and OA, and provide new pathways for the further investigation on the role of the proinflammatory cytokines in human OP and OA.

\section{Abbreviations}

OP: Osteoporosis; OA: Osteoarthritis; BMD: Bone mineral density; BTM: Bone turnover markers; RANK: Receptor activator of nuclear factor KB; RANKL: RANK ligand; OPG: Osteoprotegerin; IL-: Interleukin-; IFN-: Interferon-; TNF-a: Tumor necrosis factor-a; TGF- $\beta 1$ : Transforming growth factor $\beta 1$; CALCR:
Calcitonin receptor gene; ITGB3: $\beta_{3}$ integrin gene; OSCAR: Osteoclast associated immunoglobulin-like receptor; CTSK: Cathepsin K gene; TRAP: Tartrate resistant acid phosphatase; ACP5: Tartrate resistant acid phosphatase gene.

\section{Acknowledgements}

The authors acknowledge D. Bitenc Logar and J. Dragojevic for advice on mRNA expression measurement, I. Locatelli for advice on statistical methods, Professor R. Pain for reviewing the manuscript, and the patients participating in the study. The study was financially supported by research project J32330 of the Slovenian Research Agency which had no other involvement in the study.

\section{Author details}

${ }^{1}$ University of Ljubljana, Faculty of Pharmacy, Department of Clinical Biochemistry, Askerceva cesta 7, SI-1000 Ljubljana, Slovenia. ${ }^{2}$ General Hospital Celje, Department of Traumatology, Oblakova 5, SI-3000 Celje, Slovenia.

\section{Authors' contributions}

JZ conceived of the study, performed the gene expression analysis, analysed the data and drafted the manuscript; RK organized the collecting of the human bone and blood samples and the patients' data, JM supervised the whole study from the beginning to the accomplished manuscript preparation. All authors read and approved the final manuscript.

\section{Competing interests}

The authors declare that they have no competing interests.

Received: 2 November 2011 Accepted: 1 March 2012

Published: 1 March 2012

\section{References}

1. Lee SH, Kim TS, Choi Y, Lorenzo J: Osteoimmunology: cytokines and the skeletal system. BMB Rep 2008, 41(7):495-510.

2. Gao $Y$, Grassi F, Ryan MR, Terauchi M, Page $K$, Yang $X$, Weitzmann MN, Pacifici R: IFN-gamma stimulates osteoclast formation and bone loss in vivo via antigen-driven T cell activation. J Clin Invest 2007, 117(1):122-132.

3. Lencel $P$, Magne D: Inflammaging: The driving force in osteoporosis? Med Hypotheses 2010, 76(3):317-321.

4. Bondeson J, Blom AB, Wainwright $S$, Hughes $C$, Caterson B, van den Berg WB: The role of synovial macrophages and macrophage-produced mediators in driving inflammatory and destructive responses in osteoarthritis. Arthritis Rheum 2010, 62(3):647-657.

5. Pino AM, Rios S, Astudillo P, Fernandez M, Figueroa P, Seitz G, Rodriguez JP. Concentration of adipogenic and proinflammatory cytokines in the bone marrow supernatant fluid of osteoporotic women. J Bone Miner Res 2010, 25(3):492-498.

6. Pacifici $R$, Brown C, Puscheck E, Friedrich E, Slatopolsky E, Maggio D, McCracken R, Avioli LV: Effect of surgical menopause and estrogen replacement on cytokine release from human blood mononuclear cells. Proc Natl Acad Sci USA 1991, 88(12):5134-5138.

7. Pfeilschifter J, Koditz R, Pfohl M, Schatz H: Changes in proinflammatory cytokine activity after menopause. Endocr Rev 2002, 23(1):90-119.

8. Weitzmann MN, Pacifici R: Estrogen deficiency and bone loss: an inflammatory tale. J Clin Invest 2006, 116(5):1186-1194.

9. Cauley JA, Danielson ME, Boudreau RM, Forrest KY, Zmuda JM, Pahor M, Tylavsky FA, Cummings SR, Harris TB, Newman AB: Inflammatory markers and incident fracture risk in older men and women: the Health Aging and Body Composition Study. J Bone Miner Res 2007, 22(7):1088-1095.

10. Scheidt-Nave C, Bismar H, Leidig-Bruckner G, Woitge H, Seibel MJ, Ziegler R, Pfeilschifter J: Serum interleukin 6 is a major predictor of bone loss in women specific to the first decade past menopause. J Clin Endocrinol Metab 2001, 86(5):2032-2042.

11. Charatcharoenwitthaya N, Khosla S, Atkinson EJ, McCready LK, Riggs BL: Effect of blockade of TNF-alpha and interleukin-1 action on bone resorption in early postmenopausal women. J Bone Miner Res 2007, 22(5):724-729.

12. Takayanagi H, Kim S, Matsuo K, Suzuki H, Suzuki T, Sato K, Yokochi T, Oda H, Nakamura K, Ida N, et al: RANKL maintains bone homeostasis through cFos-dependent induction of interferon-beta. Nature 2002, 416(6882):744-749. 
13. Takayanagi H, Ogasawara K, Hida S, Chiba T, Murata S, Sato K, Takaoka A, Yokochi T, Oda H, Tanaka K, et al: T-cell-mediated regulation of osteoclastogenesis by signalling cross-talk between RANKL and IFNgamma. Nature 2000, 408(6812):600-605.

14. Cenci S, Toraldo G, Weitzmann MN, Roggia C, Gao Y, Qian WP, Sierra O, Pacifici R: Estrogen deficiency induces bone loss by increasing $T$ cell proliferation and lifespan through IFN-gamma-induced class II transactivator. Proc Natl Acad Sci USA 2003, 100(18):10405-10410.

15. Janssens $K$, ten Dijke $P$, Janssens $S$, Van Hul W: Transforming growth factor-beta1 to the bone. Endocr Rev 2005, 26(6):743-774.

16. Kudo O, Fujikawa Y, Itonaga I, Sabokbar A, Torisu T, Athanasou NA: Proinflammatory cytokine (TNFalpha/IL-1alpha) induction of human osteoclast formation. J Pathol 2002, 198(2):220-227.

17. Nakashima T, Kobayashi Y, Yamasaki S, Kawakami A, Eguchi K, Sasaki H, Sakai $\mathrm{H}$ : Protein expression and functional difference of membranebound and soluble receptor activator of NF-kappaB ligand: modulation of the expression by osteotropic factors and cytokines. Biochem Biophys Res Commun 2000, 275(3):768-775.

18. Huang W, Drissi MH, O'Keefe RJ, Schwarz EM: A rapid multiparameter approach to study factors that regulate osteoclastogenesis: demonstration of the combinatorial dominant effects of TNF-alpha and TGF-beta in RANKL-mediated osteoclastogenesis. Calcif Tissue Int 2003, 73(6):584-593.

19. Lovibond AC, Haque SJ, Chambers TJ, Fox SW: TGF-beta-induced SOCS3 expression augments TNF-alpha-induced osteoclast formation. Biochem Biophys Res Commun 2003, 309(4):762-767.

20. Wei S, Kitaura H, Zhou P, Ross FP, Teitelbaum SL: IL-1 mediates TNFinduced osteoclastogenesis. J Clin Invest 2005, 115(2):282-290.

21. Lubberts $\mathrm{E}: \mathrm{IL}-17 / \mathrm{Th} 17$ targeting: on the road to prevent chronic destructive arthritis? Cytokine 2008, 41(2):84-91.

22. Kudo O, Sabokbar A, Pocock A, Itonaga I, Fujikawa Y, Athanasou NA: Interleukin-6 and interleukin-11 support human osteoclast formation by a RANKL-independent mechanism. Bone 2003, 32(1):1-7.

23. Yoshitake F, Itoh S, Narita H, Ishihara K, Ebisu S: Interleukin-6 directly inhibits osteoclast differentiation by suppressing receptor activator of NF-kappaB signaling pathways. J Biol Chem 2008, 283(17):11535-11540.

24. Theoleyre S, Wittrant Y, Tat SK, Fortun Y, Redini F, Heymann D: The molecular triad OPG/RANK/RANKL: involvement in the orchestration of pathophysiological bone remodeling. Cytokine Growth Factor Rev 2004, 15(6):457-475

25. Fuller K, Murphy C, Kirstein B, Fox SW, Chambers TJ: TNFalpha potently activates osteoclasts, through a direct action independent of and strongly synergistic with RANKL. Endocrinology 2002, 143(3):1108-1118.

26. Itonaga I, Sabokbar A, Sun SG, Kudo O, Danks L, Ferguson D, Fujikawa Y, Athanasou NA: Transforming growth factor-beta induces osteoclast formation in the absence of RANKL. Bone 2004, 34(1):57-64.

27. Kobayashi K, Takahashi N, Jimi E, Udagawa N, Takami M, Kotake S, Nakagawa N, Kinosaki M, Yamaguchi K, Shima N, et al: Tumor necrosis factor alpha stimulates osteoclast differentiation by a mechanism independent of the ODF/RANKL-RANK interaction. J Exp Med 2000, 191(2):275-286.

28. Asagiri $\mathrm{M}$, Takayanagi $\mathrm{H}$ : The molecular understanding of osteoclast differentiation. Bone 2007, 40(2):251-264.

29. Ralston SH: Analysis of gene expression in human bone biopsies by polymerase chain reaction: evidence for enhanced cytokine expression in postmenopausal osteoporosis. J Bone Miner Res 1994, 9(6):883-890.

30. D'Amelio P, Roato I, D'Amico L, Veneziano L, Suman E, Sassi F, Bisignano G, Ferracini R, Gargiulo G, Castoldi F, et al: Bone and bone marrow proosteoclastogenic cytokines are up-regulated in osteoporosis fragility fractures. Osteoporos Int 2010, 22(11):2869-2877.

31. Hopwood B, Gronthos S, Kuliwaba JS, Robey PG, Findlay DM, Fazzalari NL: Identification of differentially expressed genes between osteoarthritic and normal trabecular bone from the intertrochanteric region of the proximal femur using cDNA microarray analysis. Bone 2005, 36(4):635-644.

32. Kuliwaba JS, Findlay DM, Atkins GJ, Forwood MR, Fazzalari NL: Enhanced expression of osteocalcin mRNA in human osteoarthritic trabecular bone of the proximal femur is associated with decreased expression of interleukin-6 and interleukin-11 mRNA. J Bone Miner Res 2000, 15(2):332-341.

33. Stewart A, Black AJ: Bone mineral density in osteoarthritis. Curr Opin Rheumatol 2000, 12(5):464-467.
34. Harris WH: Traumatic arthritis of the hip after dislocation and acetabular fractures: treatment by mold arthroplasty. An end-result study using a new method of result evaluation. J Bone Joint Surg Am 1969, 51(4):737-755.

35. Logar DB, Komadina R, Prezelj J, Ostanek B, Trost Z, Marc J: Expression of bone resorption genes in osteoarthritis and in osteoporosis. J Bone Miner Metab 2007, 25(4):219-225.

36. Kamolmatyakul S, Chen W, Li YP: Interferon-gamma down-regulates gene expression of cathepsin $\mathrm{K}$ in osteoclasts and inhibits osteoclast formation. J Dent Res 2001, 80(1):351-355.

37. Findlay DM, Atkins GJ: Relationship between serum RANKL and RANKL in bone. Osteoporos Int 2011, 22(10):2597-2602.

38. Yan T, Riggs BL, Boyle WJ, Khosla S: Regulation of osteoclastogenesis and RANK expression by TGF-beta1. J Cell Biochem 2001, 83(2):320-325.

\section{doi:10.1186/1423-0127-19-28}

Cite this article as: Zupan et al:: The relationship between

osteoclastogenic and anti-osteoclastogenic pro-inflammatory cytokines differs in human osteoporotic and osteoarthritic bone tissues. Journal of Biomedical Science 2012 19:28.

\section{Submit your next manuscript to BioMed Central and take full advantage of:}

- Convenient online submission

- Thorough peer review

- No space constraints or color figure charges

- Immediate publication on acceptance

- Inclusion in PubMed, CAS, Scopus and Google Scholar

- Research which is freely available for redistribution

Submit your manuscript at www.biomedcentral com/submit
Ciomed Central 